\title{
Verschuldung und Anpassung - die schwarzafrikanische Malaise
}

\author{
Von Wolfgang Zehender
}

Man mag mit dem Wort Krise oft leicht bei der Hand sein, aber die bedrückenden Lebensverhältnisse in vielen Ländern der Dritten Welt lassen derzeit kaum eine andere Beschreibung zu. Besonders ausgeprägt ist die Entwicklungskrise in Schwarzafrika. Zwar zeichnen sich die Länder südlich der Sahara durch große Unterschiedlichkeit im politischen System wie in der natürlichen Ressourcenausstattung, im sozio-kulturellen Gefüge wie in der Wirtschaftsordnung aus; dennoch zeigen - von wenigen Ausnahmen abgesehen - die Länder ähnliche Symptome.

Dabei herrscht unter Analytikern weitgehend Einigkeit über das Ausmaß der Krisensituation und die wirtschaftlichen und sozialen Indikatoren, anhand derer sie dargestellt werden kann. Einigkeit herrscht auch über die Ursachen dieser Krise, nicht hingegen über das Gewicht, das einzelnen Ursachenbündeln zugemessen wird. Entsprechend umstritten sind die Vorstellungen über die Maßnahmen, mit denen der Krise begegnet werden soll.

\section{Die wirtschaftliche und soziale Lage}

Die schwarzafrikanischen Staaten haben in ihrer nach-kolonialen Geschichte nur ein Jahrzehnt lang, bis zur Mitte der siebziger Jahre, nennenswerte positive Wachstumsraten ihres Bruttosozialprodukts (BSP) pro Kopf erlebt. Bereits mit dem ersten Olpreisboom ist das Wachstum im Durchschnitt auf unter $1 \%$ gefallen; seit dem zweiten Preissprung an den Weltrohölmärkten 1979/80 sind diese Raten negativ (vgl. Tabelle 1). ${ }^{1}$ Das reale BSP pro Kopf ist demnach im Jahre 1988 wieder auf das Niveau der Jahre 1968/69 zurückgefallen.

Noch einschneidender für die Lebensverhältnisse der Bevölkerung ist ein anderer Indikator, die Nahrungsmittelproduktion. Schwarzafrika ist die einzige Entwicklungsländerregion, in der die Produktion von Nahrungsmitteln pro Kopf im Trend in den vergangenen 25 Jahren zurückgegangen ist. Die Folgen sind nicht nur an der Mangel- und Fehlernährung abzulesen, sondern auch an dem wachsenden Bedarf an Nahrungsmittelimporten: Mitte der achtziger Jahre lag dieser Import bei rund 10 Mio. Tonnen mit steigender Tendenz. ${ }^{2}$

1 Soweit nicht anders angegeben, stammen die Zahlen von der Weltbank (Weltentwicklungsbericht).

2 Vgl. dazu beispielsweise die Studie von G. Geier, Nahrungsmittelhilfe und Ernährungssicherungsprogramme als Instrument der Entwicklungszusammenarbeit, DIE, Berlin 1988. 


\begin{tabular}{|lcccc|}
\hline Tabelle 1 - $\begin{array}{l}\text { Wachstumsraten von realem Bruttosozial- } \\
\text { produkt und Bevölkerung in Schwarzafrika }\end{array}$ \\
\hline & $1965-73$ & $1973-80$ & $1980-86$ & 1987 \\
\hline BSP & 6,4 & 3,5 & 0,2 & $-1,4$ \\
Bevölkerung & 2,7 & 2,8 & 3,1 & 3,2 \\
BSP pro Kopf & 3,7 & 0,7 & $-2,9$ & $-4,6$ \\
\hline
\end{tabular}

Auch die Leistung des Industriesektors ist im Durchschnitt der Länder in den Jahren 1980 bis 1986 um jährlich 2,4 \% gefallen. Das gleiche gilt für den Export von Rohstoffen. Wie das statistische Zahlenbild zeigt, ist im Durchschnitt der achtziger Jahre nicht nur das Exportvolumen zurückgegangen, sondern auch die dafür erlösten Preise, was indirekt an den massiv gefallenen terms of trade abgelesen werden kann (vgl. Tabelle 2).

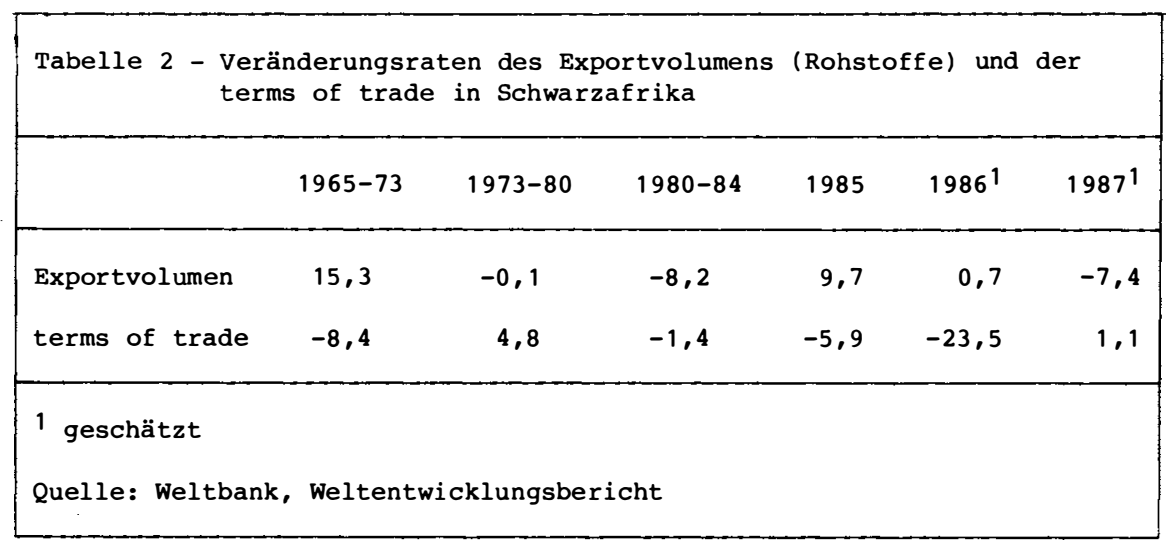

Der Druck auf die Exporterlöse und die zunehmenden Nahrungsmittelimporte mit den daraus resultierenden Handelsbilanzdefiziten, aber auch die teilweise leichtfertig aufgenommenen Kredite für nicht immer produktive Vorhaben haben die Verschuldung Schwarzafrikas in die Höhe getrieben. Ende 1987 betrug die Auslandsverschuldung der 44 Staaten Afrikas südlich der Sahara knapp 140 Mrd. US \$. ${ }^{3}$ Problematisch erscheint

3 Die Verschuldungsziffern stammen aus IMF, World Economic Outlook. 
dabei weniger die absolute Höhe dieser Summe, sie entspricht lediglich einem Zehntel der Auslandsverschuldung der Dritten Welt. Die Brisanz dieser Zahlen ergibt sich erst aus dem Vergleich zur Leistungsfähigkeit der Länder:

- gemessen an den Exporten erreicht die Verschuldung im Durchschnitt der Länder rund $325 \%$ und liegt mit dieser Quote auf gleicher Höhe mit der der fünfzehn am höchsten verschuldeten Entwicklungsländer;

- gemessen am Bruttoinlandsprodukt beträgt der Verschuldungsgrad rund $70 \%$ und übersteigt den entsprechenden Grad der fünfzehn Vergleichsländer um ein Beträchtliches;

- zur Schuldenbedienung (Zinsen und Tilgung) müssen die schwarzafrikanischen Länder rund ein Viertel ihrer Exporterlöse aufwenden.

Es liegt auf der Hand, daß sich hinter diesen Durchschnittszahlen erhebliche Unterschiede von Land zu Land verbergen. Allein vier Länder zeigen eine Quote von Schulden zu Export von rund $1500 \%$ oder darüber (Guinea-Bissau, Somalia, Sudan und Mosambique), und auch die Verschuldung Burundis, Madagaskas, Tanzanias und Zambias hat relativ besorgniserregende Höhen erreicht. Die relative Schuldensituation erklärt auch, daß nur 12 der 44 Länder die Jahre 1980 bis 1987 ohne Zahlungsrückstände bzw. Umschuldungen überstanden haben.

Angesichts der sich verschlechternden wirtschaftlichen Situation überrascht nicht mehr, daß sich auch die soziale Lage der Mehrheit der Bevölkerung in Schwarzafrika verschlimmert. Die Datenverfügbarekit in diesem Bereich ist lückenhaft; zudem erschließen sich eine Reihe von einschlägigen Sachverhalten nicht der quantitativen Erfassung, schon gar nicht über 44 Länder hinweg. Dennoch ist der Tendenz nach festzustellen: ${ }^{4}$

- eine Verschlechterung des Gesundheitszustandes,

- eine Erhöhung der Kindersterblichkeit,

- größere Teile der Bevölkerung, die fehlernährt sind,

- ein Ansteigen der Arbeitslosigkeit,

- ein Anstieg der Zahl der absolut Armen.

Insgesamt sehen sich die Staaten südlich der Sahara wachsendem wirtschaftlichem und sozialem Druck bei geringer werdenden außen- und binnenwirtschaftlichen Handlungsspielräumen ausgesetzt.

\section{Die Ursachen für diese Fehlentwicklung}

Schwarzafrika ist in seinen Entwicklungsaussichten grundsätzlich beeinträchtigt durch eine Reihe von Hemmnissen, die weder durch die Staaten selbst noch durch externe Ak-

4 Vgl. als Quellen beispielsweise die Weltbank-Publikationen Poverty and Hunger: Issues and Options for Food Security in Developing Countries, Washington, D.C. 1986, L. Demery, T. Addison, The Alleviation of Poverty under Structural Adjustment, Washington, D.C. 1987, oder auch G. Lachenmann, Anmerkungen zu den gesellschaftlichen Aspekten der Strukturkrise in Afrika, DIE, Berlin 1987. 
teure beeinflußt, geschweige denn beseitigt werden können. Dazu gehört seine koloniale Vergangenheit mit dem Erbe eines ungenügenden Ausbildungsstandes auf allen Ebenen, einer auch heute noch eher den Industrieländerinteressen als den eigenen Entwicklungsnotwendigkeiten entgegenkommenden Verkehrs- und Kommunikationsinfrastruktur und einer diesem Interesse entsprechenden Struktur der verarbeitenden Industrie; auch viele der gerade in Afrika häufigen Bürgerkriege und Umstürze mit den daraus resultierenden Flüchtlingsströmen finden hier ihre Ursache. Schließlich zählen dazu die geringe Fruchtbarkeit der tropischen Böden ${ }^{5}$ und die Unsicherheit des Klimas.

Entscheidend für die Úberlegung, wo die Ansatzpunkte für die Krisenbewältigung liegen, sind jedoch zwei Ursachenbündel:

- ein externes, das sich im wesentlichen aus weltwirtschaftlichen Gegebenheiten und den Entwicklungshilfepolitiken der Geber zusammensetzt,

- ein internes, das auf die hausgemachten Fehlentwicklungen abstellt.

Beide sind ausreichend dokumentiert und diskutiert, auch von den betroffenen schwarzafrikanischen Staaten selbst. ${ }^{6}$

\section{Weltwirtschaftliche Einflüsse und Geberverhalten}

Es gibt nur wenig schwarzafrikanische Volkswirtschaften, die nicht vom Export einiger weniger mineralischer und/oder agrarischer Rohstoffe abhängig sind. Die Devisenerlöse aus diesen Exporten machen im Regelfall 60 bis $90 \%$ des gesamten Exportwertes aus und bestimmen weitgehend den Importspielraum, die Erlöse aus Exportabgaben steuern bis zu $50 \%$ zu den öffentlichen Haushaltseinnahmen bei. Mit geringen Ausnahmen (beispielsweise bei strategischen Rohstoffen wie Titan, Molybdän u.ä.) handelt es sich dabei um gängige Rohstoffe, deren Preise, etwa durch Kartellierung (wie im Fall OPEC), von den afrikanischen Staaten nicht zu beeinflussen sind. Seit Mitte der siebziger Jahre sind die Rohstoffpreise einem tendenziellen Verfall ausgesetzt, der sich Mitte der achtziger Jahre beschleunigt hat. Die am häufigsten genannten Ursachen sind der Weltkonjunktureinbruch zu Beginn der achtziger Jahre mit entsprechendem Nachfragerückgang, aber auch der technische Fortschritt in den Industriestaaten, der zunehmend die Substitution bzw. die Einsparung gerade von "afrikanischen" Mineralrohstoffen erlaubt und die Nachfrage weiter dämpft. Da sich im gleichen Zeitraum von Schwarzafrika importierte Industriegüter verteuerten, kam es zu dem in Tabelle 2 wiedergegebenen Verfall der Austauschverhältnisse. Es ist wohl kaum allzu spekulativ, anzunehmen, daß ein ver-

5 Vgl. dazu etwa P. Waller, The Ecological Handicap of the Tropics, in: Intereconomics, May/June 1984.

6 Aus der Flut an Literatur vgl. insbesondere H. Brandt et al., Afrika in Bedrängnis, Entwicklungskrise und Neugestaltung der Entwicklungspolitik, Reihe Problem Nr. 15, Deutsche Welthungerhilfe, Bd. I und II, Bonn 1986; Stragegies for African Development, Hrsg.: R. J. Berg, J. S. Whitaker, Berkeley 1986; Africa in Economic Crisis, Hrsg.: J. Ravenhill, London 1986; UN Economic Commission for Africa, Beyond Recovery: ECA-Revised Perspectives of Africa's Development, 1988-2008, Addis Abeba 1988. 
gleichbarer Verfall der terms of trade eine europäische Volkswirtschaft in eine tiefe wirtschaftliche Rezession geführt hätte.

Die wenigen Staaten südlich der Sahara, die über einen wettbewerbsfähigen Industriesektor verfügen, haben zwar den Vorteil, tendenziell bessere Preise für ihre Industriegüter durchsetzen zu können, sehen sich allerdings zunehmenden Handelsbarrieren in den Industrieländern gegenüber. Diese Aussage klingt paradox für die durch den Vertrag von Lomé geregelten Austauschbeziehungen zwischen den EG-Ländern und den mittlerweile 66 Entwicklungsländern Afrikas, der Karibik und des Pazifiks, der diesen Ländern im Prinzip freien Marktzugang auch für verarbeitete Produkte eröffnet. Aber die Erfahrung zeigt, daß sprunghafte Zuwachsraten beim Export besonders sensibler Produkte auf EG-Seite auf zunehmende Phantasie bei der Behinderung solcher Exporte stoßen. Auch das Verhalten der verschiedenen Geber von Entwicklungshilfe war der Entwicklung nicht immer förderlich. Ein unkoordiniertes Vorgehen hat häufig zur Einrichtung von Doppel- (auch Personal-) Strukturen geführt, Lieferbindung hat Projekte verteuert, schließlich hat die Bevorzugung immer neuer, kapazitätserweiternder Vorhaben mit ihren den Nehmern überlassenen laufenden Kosten deren Budgetspielraum weit überstrapaziert.

\section{Die Politik des "urban bias"}

Der Hinweis auf Kolonialerbe und Ressourcenknappheit, Olpreise, Weltkonjunktur und fragwürdiges Geberverhalten genügt allerdings nicht, um die derzeitige Lage und die verringerten Entwicklungsaussichten in Schwarzafrika zu erklären. Unterlassene Reformen und einseitige Begünstigung bestimmter Sektoren und Regionen haben in vielen Ländern zu Strukturverzerrungen geführt, die die Auswirkungen der externen Entwicklungen auf die wirtschaftliche und soziale Situation der Bevölkerung noch verschärfen. Die häufig massive volkswirtschaftliche Fehlsteuerung von Produktionsmitteln zugunsten des Aufbaus von städtisch-modernen Anlagen und die Durchsetzung von Politikmaßnahmen zugunsten der Bedürfnisse der städtischen Eliten ist in der Summe zu einem "urban bias" gewachsen, wie er in anderen Entwicklungsländerregionen in diesem Ausmaß nicht anzutreffen ist.

Das läßt sich am Beispiel des Agrarsektors zeigen. In nahezu allen Ländern Schwarzafrikas konnte man bis vor wenigen Jahren überhöhte Wechselkurse registrieren, was etwa an der Abweichung der offiziellen von den Schwarzmarktkursen, zumindest der Tendenz nach, abzulesen war. Dies hatte zur Folge:

- Erleichterung von Konsumimporten, die eher von städtischen als ländlichen Nachfragern vorgenommen werden,

- Erleichterung von billigen Nahrungsmittelimporten, oft zu Preisen unter den bäuerlichen Produktionskosten,

- geringere Erlöse für den Export von Agrarprodukten. 
In die gleiche Richtung des Abschöpfens von Mitteln aus dem Agrarsektor zugunsten der Bef riedigung urbaner Bedürfnisse gingen Maßnahmen wie Agrarexportsteuern, niedrige Aufkaufpreise für landwirtschaftliche Produkte, subventionierte Nahrungsmittelpreise in den Städten. Die Vernachlässigung des ländlichen Raumes auch bei der verkehrsmäßigen Erschließung, beim Aufbau von sozialer Infrastruktur und bei der Schaffung von nichtlandwirtschaftlichen Arbeitsplätzen erklärt schließlich die hohe Einkommensdisparität zwischen Stadt und Land, die zunehmende Landflucht gerade der aufgeweckteren Leute, das Vernachlässigen eines agrartechnischen Fortschritts und die in manchen Regionen zunehmende Destabilisierung der traditionellen Produktionssysteme. Der Aufbau eines immer weitverzweigteren Netzes von parastaatlichen Institutionen, deren Ziel eher die Einrichtung von Arbeitsplätzen und Pfründen als die Verbesserung der Agrarproduktion war, verstärkte diese Fehlentwicklung.

Auch die industrielle Entwicklung in Schwarzafrika hat die in sie gesetzten Hoffnungen nicht erfüllt.?

Die meisten Produktionslinien sind vergleichsweise kapitalintensiv aufgebaut worden, mit den zur Aufbauzeit gängigen Industrieländer-Technologien und den entsprechenden Produktionskapazitäten. Die industrielle Förderungspolitik, insbesondere die massive Subventionierung des Faktors Kapital, war den eigentlichen Faktorknappheitsverhältnissen nicht angemessen und hat industrielle Investitionen, die ohne Subventionen wegen der Marktenge nicht rentabel gewesen wären, erst ermöglicht. Eine Anpassung an die jeweils vorhandene spezifische Faktorausstattung hat nicht stattgefunden. Das dafür notwendige sozio-ökonomische und technisch-wissenschaftliche Milieu fehlt in den meisten Ländern, mit der Folge, daß die von außen ins Land gebrachten Technologien und Produkte ungeprüft in bezug auf die einheimischen Fähigkeiten, Faktorstrukturen und Nachfragemuster übernommen wurden.

Heute sind viele dieser Betriebsstätten nur zu 20 bis $40 \%$ ihrer ursprünglichen Kapazität ausgelastet. Zudem sind häufig vorhandene lokale Aktivitäten in Handwerk und Kleinindustrie aus dem Markt verdrängt worden. Beide Tendenzen zusammen haben das Arbeitslosenproblem erhöht. Die niedrigere Wettbewerbsfähigkeit und eine eher unbedeutende Wertschöpfung haben zu Devisenverzehr und Verschwendung öffentlicher Einnahmen, nicht zu deren Schaffung beigetragen.

\section{Die Anpassungswege von IWF und Weltbank}

Internationaler Währungsfonds und Weltbank haben bei dem Versuch, die Entwicklungskrise in Schwarzafrika zu bewältigen, eine Vorreiterrolle eingenommen. Die Bedeutung der beiden Finanzinstitute ergibt sich dabei nicht so sehr aus den besonders ho-

7 Vgl. dazu W. Zehender, Industrialisierung und regionale Kooperation in Schwarzaf rika, in: Konjunkturpolitik, 32. Jg., 1986, H. 4. 
hen Finanzmitteln, die sie der Dritten Welt zur Verfügung stellen könnten, sondern aus der Tatsache, daß die wichtigsten großen Geberländer diese Rolle akzeptieren und einen Teil ihrer eigenen Entwicklungshilfemittel, zumindest einen großen Teil der nicht-projektgebundenen, an das Zustandekommen einer Ubereinkunft zwischen IWF bzw. Weltbank und Entwicklungsland binden. Auch die meisten Geschäftsbanken akzeptieren diese Rolle; dies hat allerdings für Lateinamerika größere Bedeutung, da die Banken in Schwarzafrika nur noch geringe Kredit-Neuengagements eingehen.

Die beiden Institutionen pflegten zu Beginn bis Mitte der achtziger Jahre eine klare "Arbeitsteilung" bei der Wahl der Ansatzpunkte zur Krisenbewältigung, die durch ihre jeweiligen Statuten vorgegeben ist. Die IWF-Sichtweise ist auf die Lösung von Zahlungsbilanzproblemen ausgerichtet, die Weltbank konzentrierte sich auf die Unterstützung eher langfristig ausgelegter Entwicklungsprozesse.

\section{Kurzfristige Stabilisierung: Das IWF-Vorgehen}

Die IWF-Finanzhilfe, normalerweise mit drei bis fünf Jahren Lauf zeit und zu Marktzinsen vergeben, wird gegen die Zusage der Regierung bereitgestellt, ein Stabilisierungsprogramm in Angriff zu nehmen. Die Auszahlung der einzelnen Tranchen dieser Kredite ist an die Erfüllung bestimmter wirtschaftspolitischer Vorgaben gebunden, im Regelfall wird der Abschluß eines Programms an bereits vorab zu erfüllende Bedingungen gekoppelt.

Dem Ziel "kurzfristiger Abbau von Zahlungsbilanzungleichgewichten" gemäß setzen die IWF-Auflagen an der Dämpfung der Nachfrage in den betroffenen Volkswirtschaften an; ${ }^{8}$ dies bedeutet in erster Linie die Änderung von monetären Makro-Aggregaten. Ein für Schwarzafrika typisches Konditionenpaket würde damit etwa folgende Elemente aufweisen:

- Abwertung der Landeswährung, im Regelfall als Vorab-Auflage;

- Limitierung der Kreditaufnahme im Inland wie im Ausland;

- Beschränkung der staatlichen Haushaltsausgaben, insbesondere solche mit Subventionscharakter;

- Dämpfung von Löhnen und Zinsen und Verbesserung der Preise für landwirtschaftliche Produzenten.

Insgesamt werden von einem solchen Stabilisierungsprogramm schnelle Erfolge erwartet, insbesondere im Hinblick auf die Einschränkung von Importen und den Anstieg von Exporten. Dies setzt allerdings eine Volkswirtschaft voraus, die einer funktionierenden Marktwirtschaft gleicht, in der also keine Strukturrigiditäten die Anpassungsgeschwin-

8 Vgl. dazu beispielsweise von IWF-Direktor M. Guitián, Adjustment and Economic Growth: Their Fundamental Complementarity, in: Growth-Oriented Adjustment Programs, Hrsg.: V. Corbo, M. Goldstein, M. Khan, Washington, D.C. 1987, S. 63-94. 
digkeit hemmen, ein System, das geldwirtschaftlich weitgehend integriert ist, um auf monetäre Impulse auch ausreichend reagieren zu können. Zudem wird angenommen, daß die Regierung fest genug im Sattel sitzt, um nationale Gruppeninteressen politisch im Zaume halten zu können. ${ }^{9}$

Das diesen Programmansätzen zugrundeliegende Modell schließt auch ein, daß nach kurzer Zeit der Stabilisierung über die Nachfragedämpfung der Wachstumsprozeß wieder um so stärker und nachhaltiger einsetzt. Die Abmilderung ungewollter sozialer Folgen, den Ausgleich unangenehmer Verteilungswirkungen, die rasche politische Uberwindung von etwa doch vorhandenen Rigiditäten überläßt der IWF der nationalen Entscheidung; sie werden zwar vom IWF gesehen, nicht jedoch als Programmauftrag verstanden. ${ }^{10}$

\section{Mittelfristige Anpassung: Die Weltbank-Programme}

Die Strukturanpassungsdarlehen der Weltbank, seit 1980 vergeben als nicht-projektgebundene Kredite, sind mit Anpassungsprogrammen gekoppelt, die mit ihrer längerfristi- gen Auslegung der Tendenz nach eher an der Angebotsseite einer Volkswirtschaft ansetzen. Die Programmziele werden von der Weltbank folgendermaßen umschrieben: ${ }^{11}$

- Wiederherstellung der makroökonomischen Stabilität,

- Mobilisierung der einheimischen Ressourcen,

- Steigerung der Faktorproduktivität und der Effektivität der Ressourcenallokation,

- Umstrukturierung der produktiven Sektoren zugunsten der Produktion auch international handelbarer Güter,

- Verbesserung der Leistungsfähigkeit des öffentlichen Sektors.

Die Weltbank hält den Anspruch aufrecht, daß der Komposition der Anpassungsprogramme kein geschlossenes volkswirtschaftliches Modell zugrundeliegt, nicht zuletzt, weil die Datenlage die Konstruktion eines Modells, das die "richtige" Instrumentenmischung erlaubt, nachhaltig verhindert. ${ }^{12}$ Dennoch läßt eine Querschnittsbetrachtung der Programme eine gewisse Vorliebe für marktwirtschaftliche Lösungen erkennen; zudem durchzieht die Weltbank-Vorschläge ein großes Mißtrauen gegenüber dem Staat als Teilnehmer am wirtschaftlichen Geschehen. Schließlich wird auch von der Weltbank anerkannt, daß ihre Programme nicht verteilungsneutral sind; abgesehen davon, daß bestimmte Verteilungswirkungen sogar gewollt sind, wird auf den Ubergangscharakter von negativen Programmkosten verwiesen und auch darauf, daß Strukturanpassung auf lange Frist auch positive Verteilungseffekte mit sich bringt.

9 Zum zugrundeliegenden Modell etwa R. Green, The IMF and Stabilization in Sub-Saharan Africa: a critical review, IDS Discussion Paper, Brighton 1986.

10 Vgl. Guitián, a.a.O., S. 70, S. 87 f.

$11 \mathrm{Vgl}$. etwa C. Michalopoulos, World Bank Programs for Adjustment and Growth, in: Growth-Oriented Adjustment Programs, a.a.O., S. 15-62.

12 Vgl. C. Michalopoulos, a.a.O., S. 47. 
Wenn auch immer wieder das "Maßgeschneiderte" der Programme hervorgehoben wird, so fällt doch auf, daß Strukturanpassungsprogramme in Schwarzafrika nahezu in jedem Fall folgende Komponenten umfassen:

- in den öffentlichen Budgets Streichung von Stellen und Subventionen sowie Verbesserung der Einnahmenseite durch bessere Ausnutzung der Steuerbasis,

- Rückführung des Staatseinflusses über Privatisierung bzw. Schließung von öffentlichen Unternehmen; Institutionenhilfe zur Verbesserung der Effizienz in den verbliebenen staatlichen und halbstaatlichen Unternehmen,

- Veränderung des Preissystems zugunsten der landwirtschaftlichen Erzeuger,

- Verbesserung des Geld- und Kreditsystems, insbesondere auch hier durch Entsaatlichung der Institutionen,

- Veränderung des Anreizsystems (Steuervergünstigungen, Subventionen, sonstige staatliche Leistungen) zugunsten der produktiven Bereiche, innerhalb derer zugunsten der Exportproduzenten,

- Liberalisierung des Außenhandelsregimes, Reduzierung der Zölle und anderer quantitativer und qualitativer Export- und Importhemmnisse.

Als Fazit für die Programmvorschläge sowohl von IWF als auch Weltbank wird deutlich, daß die hausgemachten Fehlentwicklungen im Vordergrund der Reformbemühungen stehen. Externe Sachverhalte der schwarzafrikanischen Krise werden zwar von beiden Institutionen als Mitverursacher der Krise akzeptiert, es wird ihnen aber keine gleichgewichtige Bedeutung zugemessen. Aufforderungen an die Industrieländer zur Änderung von Politiken haben allenfalis deklamatorischen Charakter, etwa in den von der Weltbank jährlich herausgegebenen Weltentwicklungsberichten.

\section{Die ersten Erfahrungen mit der Strukturanpassung}

Seit 1980 bis 1987 hat die Weltbank insgesamt 51 Anpassungskredite vergeben; zählt man die ähnlich gearteten, also mit einem Umstrukturierungsprogramm gekoppelten, Sektoranpassungskredite dazu, dann sind dies sogar 121 Kredite mit einem Ausleihvolumen von rund 15,3 Mrd. US \$. Die Hälfte aller schwarzafrikanischen Länder (22 von 44) hat in diesem Zeitraum Anpassungsprogramme eingeleitet. ${ }^{13} 1988$ sind weitere Länder hinzugekommen. Da IWF und Weltbank arbeitsteilig, aber im allgemeinen gemeinsam vorgehen, läßt sich feststellen, daß ein Großteil der in Afrika heute getroffenen wirtschafts- und sozialpolitischen, aber auch innenpolitischen Entscheidungen von den beiden Institutionen massiv beeinflußt werden. Das Resultat dieser Bemühungen ist, gemessen am eigenen Anspruch, allenfalls gemischt zu beurteilen.

Am spektakulärsten waren die Fehlschläge in jenen Ländern, in denen sich Teile der Be-

13 Zahlen und Fakten sind enthalten in der Weltbank-Broschüre Lending for Adjustment: An Update, Washington, D.C., ohne Jahr (1988). 
völkerung militant gegen einzelne Programmkomponenten, zumeist Streichung der Subventionen für Grundnahrungsmittel, gewehrt haben. Beispiele dafür sind die "Maispreisrevolte" von Zambia und der "Zuckerpreisaufstand" im Sudan.

Weniger öffentlichkeitsträchtig sind die institutionsinternen Uberprüfungen. So hat die Evaluierungsabteilung der Weltbank 1986 eine erste Uberprüfung ihrer Strukturanpassungsdarlehen vorgenommen und kam bei der Untersuchung von 15 Fällen in 10 Ländern (auch außerhalb Schwarzafrikas) zu folgendem Ergebnis: ${ }^{14}$

- Keines der Länder konnte das Programm in der vorgesehenen Zeit umsetzen; in zwei Ländern wurden die Programme erst gar nicht in Angriff genommen.

- Fast alle Länder hatten Schwierigkeiten im Bereich der "verordneten" Exportsteigerung, der Handelsliberalisierung und der institutionellen Reformen.

- Die sozialen Folgen der Anpassung waren besonders in den ärmeren Entwicklungsländern (also insbesondere in Schwarzafrika) gravierend.

Auf der anderen Seite sind unzweifelhaft auch erfolgreiche Anpassungsfälle festzuhalten, unter denen Ghana und Malawi (zumindest bis 1987) herausragen. Insgesamt verweist die Weltbank darauf, daß in Schwarzafrika die "Anpassungsländer" heute ein besseres makroökonomisches Bild bieten als die Länder ohne Reformprogramm. ${ }^{15}$

Die Kritik, insbesondere an den harten Auflagen des IWF und seinen strengen, quantitativ fixierten Leistungskriterien sowie den nur kurzfristig und zu Marktbedingungen vergebenen Darlehen, hat dort bereits zu einer Änderung der Politik geführt. Mit der Einführung einer neuen Fazilität für ärmere Entwicklungsländer insbesondere in Schwarzaf rika, der "Structural Adjustment Facility" (SAF) und ihrer Erweiterung 1988 durch die "Enhanced SAF", sind zum einen die Kreditkonditionen verbessert (Zins 0,5\%) und die Rückzahlungsfristen ausgedehnt (fünf bis zehn Jahre) worden; zum anderen sind IWF und Weltbank gehalten, sich besser abzustimmen, was sich in dem gemeinsam zu formulierenden "Policy Framework Paper" für anpassungswillige Länder manifestiert.

Die Vergabe von "billigeren" Krediten durch den IWF kann allerdings auch anders interpretiert werden. Immerhin gelingt es damit den Ländern, ihre sehr viel teureren IWFKredite, aber auch Geschäftsbank-Darlehen zu Marktkonditionen, abzulösen. Dies ist angesichts des Nettoressourcentransfers von Afrika in die Geberländer und -institutionen, der in einigen Ländern schon eingesetzt hat, sich in anderen abzeichnet, insbesondere im Interesse der Geber.

Wie enttäuschend schließlich die Anpassungsbemühungen auf die Mobilisierung von zusätzlichen finanziellen Mitteln gewirkt hat zeigt Tabelle 3. Dort ist abzulesen, daß sich der reale Finanzmitteltransfer von 1980 bis 1987, auf die inzwischen gewachsene Bevölkerung umgerechnet, praktisch halbiert hat.

14 Vgl. World Bank: Structural Adjustment Lending. A First Review, Washington, D.C. 1986.

$15 \mathrm{Vgl}$. Lending for Adjustment: An Update, a.a.O. 


\begin{tabular}{|c|c|c|}
\hline & 1980 & 1987 \\
\hline "Official Development Aid" (netto) & 5,2 & 6,5 \\
\hline - Zuschüsse & 2,7 & 3,6 \\
\hline - Darlehen zu Vorzugsbedingungen & 2,5 & 2,9 \\
\hline Direktinvestitionen & 0,0 & 1,0 \\
\hline Darlehen zu Marktbedingungen & 5,5 & 2,3 \\
\hline "Anderes Kapital" & $-1,4$ & $-0,7$ \\
\hline Summe & 9,3 & 9,1 \\
\hline in Preisen von $1980^{1}$ & 9,2 & 5,9 \\
\hline pro Kopf der Bevölkerung (in US $\$$ ) & 28,0 & 14,4 \\
\hline \multicolumn{3}{|c|}{$\begin{array}{l}1 \text { Preis-Deflator aus IMF, World Economic Outlook } \\
\text { Quelle: Weltbank, Weltentwicklungsbericht }\end{array}$} \\
\hline
\end{tabular}

\section{Die Konditionalität in der Diskussion}

In nahezu allen mit der Krise in Schwarzafrika befaßten Zirkeln herrscht Einigkeit über die Notwendigkeit, die massiven Strukturverzerrungen in den meisten Ländern des Kontinents möglichst nachhaltig abzubauen. Auch die Mehrheit der Länder selbst hat auf Zusammenkünften im Rahmen der OAU und der UN Economic Commission for Africa (ECA) die eigene Verantwortung zum Abbau des "urban bias" ihrer Entwicklungspolitik akzeptiert; auch über einzelne Elemente der Anpassung herrscht zwischen der ECA und der Weltbank kein Dissens. ${ }^{16}$ Dennoch sind die verbleibenden Unterschiede noch gravierend genug.

Die massive Kritik am IWF/Weltbank-Vorgehen entzündet sich zunächst an den zugrundeliegenden Annahmen, die der schwarzafrikanischen Realität nicht adäquat sind. Bei aller Unterschiedlichkeit zeigen die betroffenen Länder große Ähnlichkeiten im Hin-

16 Vgl. dazu S. Please, The Hobbled Giant. Essays on the World Bank, Boulder and London 1984. 
blick auf Rigiditäten und eingefahrene Strukturen, auf die Fragmentiertheit ihrer Märkte, auf eine nur teilweise Integration der Bevölkerung und ihrer Lebensbereiche in die nationalen monetären Kreisläufe, auf eine schlecht funktionierende Verkehrs- und Kommunikationsinfrastruktur, auf große Lücken im Ausbildungsstand ihrer Arbeitskräfte. Insgesamt verhindern diese Faktoren ein rasches Reagieren der Volkswirtschaft auf die alleinige Änderung von wirtschaftlichen Makroaggregaten, zumal von monetären.

Wenn IWF und Weltbank gezwungen waren, die Tranchenauszahlung einiger ihrer Kredite zu unterbrechen, weil die vorher ausgemachten Leistungskriterien nicht erfüllt waren, so lag das eher an der realitätsfernen Formulierung der Kriterien als an der Unfähigkeit der Regierungen und Verwaltungen, die verlangten Auflagen auch durchzusetzen. Dies gilt um so mehr in den Fällen, in denen sich im Verlauf der Anpassungsprozesse der äußere Datenkranz (etwa durch weiteren Verfall der Rohstoffpreise) geändert hat, ohne daß dies ausreichend seinen Ausdruck in der Änderung der Anpassungsprogramme fand. ${ }^{17}$

Zudem hat sich häufig erst im Prozeß der Anpassung herausgestellt, daß Widersprüche im Zielsystem selbst steckten. Eine Untersuchung der Weltbank im Fall Malawi hat beispielsweise aufgezeigt, daß die Streichung von Düngemittelsubventionen nicht mit einer entsprechenden Erhöhung der Kreditlinien für Kleinbauern einherging. ${ }^{18}$ Ähnliche Ungereimtheiten lassen sich insbesondere in den Auflagen nach Streichung von Haushaltsausgaben bei gleichzeitiger Forderung nach mehr Leistung nachweisen. ${ }^{19}$

Besonders massiver Kritik sahen sich die beiden Institutionen wegen ihrer exportorientierten Empfehlungen sowie wegen der Vernachlässigung der sozialen Auswirkungen ihrer Programme ausgesetzt.

\section{Binnenmarkt-versus Exportorientierung}

Eine immer wiederkehrende Komponente der Strukturanpassung ist die Forderung, die Binnenmarktorientierung der Produktion möglichst rasch durch eine Weltmarktorientierung zu ersetzen. In der Landwirtschaft führt das, wenn konsequent betrieben, zur Ausdehnung des Angebots an Produkten wie Kaffee, Kakao und tropischen Früchten mit der Konsequenz, daß bei der niedrigen Preiselastitzität der Importnachf rage die Devisenerlöse tendenziell eher sinken; gleichzeitig sind die Länder zunehmend darauf ange-

17 Ausführlicher zur kritischen Auseinandersetzung mit dem Vorgehen von IWF und Weltbank beispielsweise die Sammelbände The IMF and the World Bank in Africa, Hrsg.: K.J. Harnevik, Scandinavian Institute of African Studies, Uppsala 1987; Growth-Oriented Adjustment Programs, a.a.O.; African Debt and Financing, Hrsg.: C. Lancaster, J. Williamson, Washington, D.C. 1986; und die Ausgaben von World Development, Bd. 15, 1987, H. 12 sowie IDS Bulletin, Bd. 19, 1988, H. 1.

18 Vgl. U. Lele, Structural Adjustment, Agricultural Development and the Poor: Some Observations on Malawi, Ms., Washington, D.C. 1987.

19 Vgl. dazu auch G. Lachenmann, Die gesellschaftliche Problematik der Strukturanpassungspolitik in Afrika, in: Viertel jahresberichte 1987, H. 109. 
wiesen, ihre Grundnahrungsmittel zu importieren. Auch die Fertigwarenexporte aus schwarzafrikanischen Staaten haben wegen ihrer im Vergleich etwa zu den südostasiatischen Exporteuren hohen Lohnkosten, geringen Produktqualitäten, der nur rudimentär ausgebauten institutionellen und betrieblichen Innovationskapazität sowie der schlechten Verkehrsinfrastruktur allenfalls marginale Zuwachschancen. Das Potential liegt daher in beiden Sektoren eher in der richtigen Mischung aus Binnen- und Außenorientierung und in dem als langfristige Aufgabe anzusehenden Aufbau einer entsprechenden innovativen Kompetenz.

\section{Soziale Auswirkungen der Anpassung}

Die Diskussion über die sozialen Folgen der Anpassung zeigt, daß es nicht einfach ist, Armut eindeutig auf Anpassungsmaßnahmen bzw. auf bestehende interne Strukturverzerrungen oder auf externe Einflüsse zurückzuführen. Allerdings können bestimmte sozioökonomische Gruppen, die zu den Anpassungsopf ern zu zählen sind, identifiziert werden:

- Entlassene aus dem Staatsdienst bzw. aus öf fentlichen Unternehmen,

- Bezieher niedriger Einkommen, insbesondere in den urbanen Regionen, denen Nahrungsmittel-Subventionen entzogen werden,

- die vor Einleitung von Anpassungsmaßnahmen schon als absolut arm eingeschätzten Gruppen ("Marginalisierte"), auf die die Budgetkürzungen im sozialen Sektor direkt durchschlagen.

Heftigen Widerspruch haben sich IWF und Weltbank für ihre Haltung eingehandelt, daß die negativen Auswirkungen zwar erkennbar sind, ${ }^{20}$ aber ihre Abmilderung nicht Aufgabe der Anpassungsprogramme sein kann. Immerhin hat die Diskussion, angeführt von UNICEF mit ihrer Untersuchung "Adjustment with a Human Face", ${ }^{21}$ zu einer ersten Reaktion der Weltbank geführt. Für Ghana wurde mit Hilfe einiger UN-Organisationen und finanzieller Unterstützung bilateraler Geber ein Programm zur Abfederung der sozialen Auswirkungen der Anpassung (PAMSCAD) auf die Beine gestellt. Es umfaßt eine große Zahl von zeitlich beschränkten Sozialhilfemaßnahmen, dazu Mittel für Kleinkredite, die den produktiven Bereich direkt stärken sollen. Die eigentlich notwendige Aufnahme von armutsorientierten Elementen in die Programme selbst ist allerdings noch nicht erkennbar.

20 Vgl. z.B. The Implications of Fund-Supported Adjustment Programs for Poverty. Experiences in selected countries. IMF Occ. Paper No. 58, Washington, D.C. 1988; Protecting the Poor During Periods of Adjustment, World Bank, Washington, D.C. 1987.

21 Vgl. Adjustment with a Human Face, Hrsg.: G. Cornia, R. Jolly, F. Stewart, Oxford 1987. 


\section{Ausblick}

Schwarzafrika wird sich wohl auf ein weiteres Jahrzehnt der Anpassung einrichten müssen. Auch die Weltbank geht davon aus, daß sie bis auf weiteres ein Viertel ihres Ausleihvolumens für die nicht-projektgebundene Anpassungsfinanzierung bereitstellen muß. Es herrscht auch Einigkeit über die Richtung: Abbau der Subventionierung der urbanen Sektoren zugunsten eines höheren Ressourcenflusses in den ländlich/landwirtschaftlichen Raum und zugunsten einer produktiveren Verwendung der Mittel. Eine derartige Anpassung benötigt jedoch angesichts der wirtschaftlichen, sozialen und politischen Realitäten in den Ländern sowie der begrenzten Aufnahmefähigkeiten der Weltmärkte einen langen Atem.

Daraus ergeben sich drei Konsequenzen für die Ausgestaltung von Anpassungsprogrammen. Erstens sind kurzf ristige Maßnahmen, womöglich mit schockartigem Ausmaß wie beispielsweise Halbierung der Wechselkurse, nicht adäquat; kein Dissens herrscht über die Notwendigkeit von Preis- und Wechselkursveränderungen, aber eine behutsamere Anwendung hat in der Regel nachhaltigere Wirkung. Zweitens kommt die Konzentration auf die Änderung von Makroaggregaten allein der Realität der inflexiblen und fragmentierten Märkte in Afrika nicht entgegen. Reformen sollten an Teilmärkten bzw. Sektoren und/oder Regionen ansetzen; dabei wird die zusätzliche Unterstützung der Regierungen beim weiteren Ausbau (nicht Abbau) der Umsetzungskapazitäten auch auf den unteren Ebenen wie beim Aufbau der innovatorischen Kompetenz nötig. Drittens müssen Konditionalität und Leistungskriterien der Anpassung flexibler gehandhabt werden; nur ein ständiges "monitoring" der Programme ermöglicht Änderungen, die sich aus der Veränderung etwa weltwirtschaftlicher Signale ergeben.

Darüber hinaus legen es die Anpassungserfahrungen der vergangenen Jahre nahe, folgende Faktoren für den Erfolg einzukalkulieren:

- Anpassungsprogramme haben auch tiefgreif ende strukturelle Veränderung der Gesellschaft zur Folge und können daher nur mit überzeugten Regierungen verwirklicht werden; dabei hilft es natürlich, wenn die regierenden Eliten nicht nur den eigenen Vorteil im Sinn haben, sondern auch entwicklungspolitisches Engagement zeigen, was wiederum besonders unterstützt werden könnte. Das beste Beispiel hierfür ist Ghana. ${ }^{22}$

- Die breite und nachhaltige Absicherung von Anpassungsbemühungen muß in Afrika, wo große Teile der Bevölkerung am Rande des Existenzminimums leben und eine weitere Verschlechterung ihrer Lage nicht hinnehmen (können), durch die aktive Berücksichtigung der sozialen Auswirkungen erfolgen. Das Warten auf die langfristigen Wirkungen des Marktes ist dabei ebenso fatal wie die Beschränkung auf Sozialhilfemaßnahmen. Vielmehr sollten armutsorientierte Elemente von vornherein in die Anpassungsprogramme eingebaut werden.

22 Den Fall Ghana, insbesondere das Engagement der Regierung Rawlings, schildert T. Siebold, Ghana 1957-1987. Entwicklung und Rückentwicklung, Verschuldung und IWF-Intervention, Hamburg 1988. 
Die Konstruktion von IWF und Weltbank macht es den beiden Institutionen leichter, Anpassung von den hochverschuldeten Entwicklungsländern zu verlangen als dies gleichzeitig auch von den Industrieländern zu fordern. Der Erfolg der afrikanischen Bemühungen hängt allerdings auch von deren Verhalten ab. Dazu gehört zunächst eine Einschränkung der protektionistischen Tendenzen, um die eher bescheidenen Lief ermöglichkeiten aus diesen Ländern nicht noch zusätzlich zu beeinträchtigen. Dazu gehört auch das massive und mittelfristige finanzielle Engagement der Geber; wie weit man davon noch entfernt ist, zeigen die Zahlen von Tabelle 3, die Rückgang statt Wachstum signalisieren. Am Ende steht die derzeit dringlichste Frage. Die Forderung nach Mobilisierung der Ressourcen in Schwarzafrika kann nicht bedeuten, den Ertrag dieser Mobilisierung zur Schuldenbedienung nach Europa oder Amerika zu überweisen. Nicht zuletzt um den peinlichen Eindruck eines auch aus einigen Ländern Schwarzafrikas zunehmenden Nettoressourcenflusses in die falsche Richtung zu korrigieren, ist eine großzügige Schuldenregelung auch mit fallweisem Schuldenverzicht die wichtigste anstehende Entscheidung. 
Indebtedness and Adjustment - the Black African Malaise

\section{By Wolfgang Zehender}

The present economic and social crisis in most Black African countries can be attributed to external as well as internal causes. Externally, the dramatic deterioration of the terms of trade has contributed to the growing indebtedness and has reduced the scope for imports necessary to maintain productive capacities. Internally, a policy of "urban bias" has led to considerable structural distortions. IMF and World Bank have taken the lead in trying to overcome the crisis by offering financial support to countries willing to start a stabilization and adjustment programme. Their prescriptions presuppose, however, an integrated economy, free of structural rigidities, strong governments committed to development, and a population which in its majority is sufficiently well off to bear a large part of the cost. Although some countries have indeed made progress, the outcome of the adjustment efforts on the whole is not very satisfactory. Objectives, ill-defined at times, have not been attained; the conditionality of programmes has proved to be too harsh, too macro-oriented and too short-term to be implemented in the inflexible, fragmented African markets; finally the vulnerability of the poor has been underestimated.

IMF and World Bank have started to change conditions and programmes: the IMF has introduced the Structural Adjustment Facility with concessionary terms, the World Bank has embarked upon a programme of mitigation of the social consequences of adjustment. However, four prerequisites have yet to be met to make adjustment more successful: a longer-term view, more flexibility and closer monitoring of progress to be able to take into account changing external conditions, the integration of poverty-oriented elements into the adjustment programmes, and the guaranteeing of a real increase in resource flows. The action most urgently to be taken, however, is a generous debt agreement and a case-by-case debt cancellation.

\section{Subtle Nuances in Multilateral Financial Aid}

\section{By Jerzy Kranz}

The article is concerned with the relations of Marxist oriented countries with the International Monetary Fund (IMF) and the World Bank Group. After a short historical introduction the author discusses relations of European socialist states and the People's Republic of China with the above-mentioned organizations. These relations do not include many conflicts and both organizations display relative realism in the evaluation of the bases and effectiveness of economic policies of the countries in question. 